\title{
Complicações locais no cateterismo venoso periférico em neonatos: coorte prospectiva
}

Mitzy Tannia Reichembach Danski ${ }^{1}$, Jolline Lind ${ }^{2}$, Priscila Mingorance ${ }^{3}$, Derdried Athanasio Johann ${ }^{4}$, Alessandra Amaral Schwanke ${ }^{5}$

\footnotetext{
${ }^{1}$ Enfermeira, Doutora em História. Professora Associada da Universidade Federal do Paraná (UFPR). Curitiba, PR, Brasil. E-mail: mitzyr257@gmail.com.

${ }^{2}$ Enfermeira. Discente do Programa de Pós-Graduação em Enfermagem, nível Mestrado, da UFPR. Curitiba, PR, Brasil. Email: lind.jolline@gmail.com.

${ }^{3}$ Enfermeira, Mestre em Enfermagem. Enfermeira da Secretaria Municipal de Saúde de Colombo. Colombo, PR, Brasil. Email:primingo@yahoo.com.br.

${ }^{4}$ Enfermeira, Doutora em Enfermagem. Enfermeira do Instituto Federal do Paraná, Campus Curitiba. Curitiba, PR, Brasil. Email: derdried.johann@ifpr.edu.br.

${ }^{5}$ Enfermeira. Discente do Programa de Pós-Graduação em Enfermagem, nível Mestrado, da UFPR. Auxiliar de Enfermagem do Hospital de Clínicas da UFPR. Curitiba, PR, Brasil. E-mail: ale_schwanke@hotmail.com.
}

Recebido: 24/03/2015.

Aceito: 24/09/2015.

Publicado: 31/03/2016.

\begin{abstract}
RESUMO
Objetivou-se avaliar incidência de complicações relacionadas ao uso do primeiro CIP em neonatos e identificar fatores de risco associados. Trata-se de coorte observacional prospectiva, com abordagem quantitativa; desenvolveu-se em Unidade de Terapia Intensiva Neonatal de hospital de ensino de uma capital do Sul do Brasil; e coleta de dados ocorreu de fevereiro a junho de 2013. Totalizou-se 134 CIP. A incidência de complicações foi de $41,01 \%$. O sexo feminino $(p=0,0152)$ apresentou menor risco de desenvolver complicação e nas primeiras 48 horas após punção ocorre maior risco de desenvolver complicação $(p=0,0296)$. As evidências resultantes desta pesquisa embasam um cuidado de qualidade, beneficiando a população em estudo.

Descritores: Enfermagem Neonatal; Tecnologia; Pesquisa em Enfermagem Clínica; Cateterismo Venoso Periférico; Complicações.
\end{abstract}

\section{INTRODUÇÃO}

A Unidade de Terapia Intensiva Neonatal (UTIN), constitui ambiente terapêutico adequado para tratamento de neonatos de alto risco ${ }^{(1)}$. Atribui ao cuidado dessa clientela o uso de equipamentos diversificados, equipe capacitada, protocolos específicos e tecnologia de ponta.

Uma das atividades realizadas diariamente nestas unidades é a terapia intravenosa (TIV) ${ }^{(2)}$. Para implementá-la, a equipe de enfermagem necessita de cateteres intravasculares, caracterizados como tecnologia dura, e são amplamente utilizados ${ }^{(2-3)}$.

Os cateteres podem ser classificados como centrais e periféricos, conforme localização da ponta do 
dispositivo intravenoso. Estudo realizado no estado do Rio de Janeiro comprovou que $99,6 \%$ dos neonatos utilizaram a via venosa durante sua internação em UTIN e que destes, 49,2\% foram cateteres intravenosos periféricos $(\mathrm{CIP})^{(4)}$. Outros estudos reforçam a ampla utilização deste dispositivo na terapêutica de pacientes internados em estabelecimentos de saúde ${ }^{(5-6)}$.

O CIP tem como indicações a administração de fluídos e eletrólitos, nutrição parenteral, medicações e coleta de sangue para exames. Considerado procedimento invasivo, apresenta menor risco quando comparado aos cateteres centrais no que se refere à ocorrência de infecções ${ }^{(3)}$.

Embora a TIV proporcione benefícios aos neonatos, inúmeras são as preocupações relacionadas a sua prática, devido aos riscos de complicações a que os pacientes estão expostos ${ }^{(3)}$. As complicações associadas a TIV classificam-se conforme a dimensão dos seus efeitos em sistêmicas e locais.

As sistêmicas são aquelas que oferecem risco à vida do paciente, como septicemia, sobrecarga circulatória, edema pulmonar, embolia gasosa. As locais caracterizam-se por lesões ao redor do local de punção e são passíveis de reconhecimento por meio de observação direta do local ${ }^{(7)}$. Os exemplos incluem: hematoma, trombose, flebite, tromboflebite, infiltração, extravasamento e infecção local ${ }^{(8-11)}$.

Estudo realizado em UTIN destaca ocorrência de complicações concernente à administração medicamentosa e relacionadas aos cateteres intravasculares ${ }^{(12)}$. Outro estudo realizado no mesmo cenário aponta complicações locais como motivo de retirada do CIP em $48 \%$ dos neonatos, reforçando o papel da enfermagem ao evitar complicações e reduzir os riscos a saúde ${ }^{(10)}$.

Há escassez de publicações nacionais acerca das complicações locais da TIV relacionadas ao uso do primeiro CIP em neonatos, fato que estimulou o desenvolvimento deste estudo.

Desta forma, com base no exposto, objetivou-se avaliar a incidência de complicações locais relacionadas ao uso do primeiro CIP em neonatos, bem como identificar os fatores de risco associados.

\section{MÉTODO}

Estudo de coorte observacional prospectivo, realizado em UTIN de um hospital de ensino de uma capital do Sul do Brasil. Os participantes da pesquisa foram todos os neonatos internados na UTIN submetidos à punção do primeiro CIP, no período de coleta de dados (1ㅇ de fevereiro a 30 de junho de 2013). Os neonatos que apresentaram fragilidade da rede venosa que impossibilitou a punção com CIP foram excluídos da pesquisa.

A coleta de dados ocorreu diariamente, de forma ininterrupta, na presença de duas pesquisadoras, mediante leitura das informações contidas nos registros do prontuário do neonato e observação direta do dispositivo, utilizando-se de instrumento próprio, elaborado com questões fechadas que abordavam dados sociodemográficos e clínicos; e relacionados à inserção, manipulação e retirada do cateter.

Com o intuito de padronizar a coleta de dados, a equipe de pesquisadores passou por capacitação diária, lado a lado, orientado pela mestranda responsável pela pesquisa, num período de três meses. Os pesquisadores participaram de reuniões, onde os conceitos foram padronizados com base na literatura 
científica. A punção e a manipulação dos dispositivos foram realizadas pela equipe de enfermagem da unidade, capacitada para tal procedimento. Os neonatos foram acompanhados desde o momento da internação até desfecho de sua internação na UTIN (alta, transferência ou óbito).

Os dados foram digitados em planilhas eletrônicas do programa Microsoft Excel ${ }^{\circledR}$ e analisados com auxílio do programa Bioestat ${ }^{\circledR}$. A variável resposta da pesquisa (ou desfecho) foi a ocorrência de complicações. $\mathrm{Na}$ análise de fatores que possam interferir na variável resposta, foram utilizados o teste do Qui-quadrado e o teste $\mathrm{G}$ de Williams para as variáveis explicativas categóricas (sexo, tipo de parto, motivo de internação, Apgar, Parkin, peso ao nascer, membro puncionado, profissional que realizou o procedimento, tempo de permanência) e o teste $U$ de Mann-Whitney para as variáveis explicativas quantitativas. Em todos os testes utilizou-se nível de significância de 5\%. Aplicou-se cálculo do risco relativo (RR) para medir o grau de associação. A categoria considerada como referência está indicada nas tabelas de resultados com o valor 1,0 , na coluna para os valores de RR.

O projeto foi aprovado pelo Comitê de Ética da instituição, conforme parecer no 165.675, sendo respeitados os aspectos éticos que envolvem pesquisas com seres humanos, de acordo com a legislação brasileira. Os pais ou responsáveis assinaram o Termo de Consentimento Livre e Esclarecido, autorizando a participação dos neonatos no estudo.

\section{RESULTADOS}

Durante a pesquisa foram observadas e registradas a inclusão de 134 cateteres. Destes, 79 (58,96\%) não desenvolveram complicações e 55 (41,04\%) desenvolveram. Dos 79 cateteres que não desenvolveram complicações, 28 (20,90\%) tiveram retiradas eletivas; oito (5,97\%) retirada acidental; e 43 (32,09\%) casos não informados.

A complicação mais predominante foi a infiltração/extravasamento representando 76,36\% (n=42) dos casos, 16,36\% ( $n=9)$ dos cateteres foram retirados devido a obstrução e 7,27\% (n=4) por flebite.

Quanto aos fatores de risco associados a complicações no uso do CIP, relacionados ao neonato, evidenciou-se significância estatística para a variável sexo, apontando que neonatos do sexo feminino apresentam menor risco de desenvolver complicação no CIP ( $p=0,0152 ; R R=0,60)$ (Tabela 1).

Ao analisar a distribuição dos cateteres com complicação de acordo com o sexo do neonato, percebese que $25(45,40 \%)$ cateteres utilizados em neonatos do sexo masculino foram retirados devido à infiltração/extravasamento, cinco $(9,10 \%)$ devido a obstrução e quatro $(7,20 \%)$ devido a flebite (Figura 1 ).

Ao relacionar os fatores de risco associados ao uso do cateter procedeu-se a análise dos dados mediante agrupamento e comparação entre cateteres com presença $(n=55)$ ou ausência de complicação $(n=79)$. Evidenciou-se maior risco de desenvolver complicação nas primeiras 48 horas após punção $(p=0,0296 ; R R=1,69)$ (Tabela 2). Não houve associações significativas com as demais variáveis analisadas.

A infiltração foi o motivo de retirada dos cateteres mais prevalente $65,45 \%(n=36)$, houve cinco $(9,09 \%)$ cateteres retirados por obstrução e um (1,80\%) por flebite antes de 48 horas (Figura 2). 
Tabela 1: Análise das variáveis dos neonatos associadas à ocorrência de complicações pelo uso do cateter intravenoso

\begin{tabular}{|c|c|c|c|c|c|c|c|}
\hline \multirow{3}{*}{ Variável } & \multicolumn{4}{|c|}{ Complicação } & \multirow{3}{*}{ p-valor } & \multirow{3}{*}{$\mathbf{R R}$} & \multirow{3}{*}{ IC [95\%] } \\
\hline & \multicolumn{2}{|c|}{ Sim } & \multicolumn{2}{|c|}{ Não } & & & \\
\hline & $\mathbf{N}$ & $\%$ & $\mathbf{N}$ & $\%$ & & & \\
\hline Sexo & $n=55$ & & $\mathrm{n}=79$ & & & & \\
\hline Feminino & 21 & 38,18 & 47 & 59,49 & 0,01521 & 0,6 & {$[0,39 ; 0,92]$} \\
\hline Masculino & 34 & 61,82 & 32 & 40,51 & & 1 & \\
\hline Tipo de parto & $n=55$ & & $n=79$ & & & & \\
\hline CST & 42 & 76,36 & 58 & 73,42 & $0,6999^{1}$ & 1,1 & {$[0,68 ; 1,78]$} \\
\hline PV & 13 & 23,64 & 21 & 26,58 & & 1 & \\
\hline Motivo de internação & $n=55$ & & $\mathrm{n}=79$ & & & & \\
\hline Prematuridade & 24 & 43,64 & 35 & 44,3 & 0,93901 & 1,03 & {$[0,81 ; 1,31]$} \\
\hline Outros & 31 & 56,36 & 44 & 55,7 & & 1 & \\
\hline Apgar1' & $n=51$ & & $\mathrm{n}=77$ & & & & \\
\hline$<7$ & 19 & 34,55 & 24 & 30,38 & 0,47541 & 1,17 & {$[0,76 ; 1,81]$} \\
\hline$\geq 7$ & 32 & 58,18 & 53 & 67,09 & & 1 & \\
\hline Apgar5' & $\mathrm{n}=51$ & & $n=77$ & & & & \\
\hline$<7$ & 4 & 7,27 & 4 & 5,06 & $0,5612^{2}$ & 1,28 & {$[0,62 ; 2,64]$} \\
\hline$\geq 7$ & 47 & 85,45 & 73 & 92,41 & & 1 & \\
\hline Parkin & $n=55$ & & $n=76$ & & & & \\
\hline$<36$ & 31 & 56,36 & 49 & 62,03 & 0,47541 & 0,82 & {$[0,76 ; 1,81]$} \\
\hline$>36$ & 24 & 43,64 & 27 & 34,18 & & 1 & \\
\hline Peso ao nascer & $n=78$ & & $n=54$ & & & & \\
\hline$<1500$ & 17 & 21,52 & 11 & 20 & 0,93001 & 0,98 & {$[0,56 ; 1,71]$} \\
\hline$>2500$ & 36 & 45,57 & 24 & 43,64 & & 1 & - \\
\hline $1500<p>2500$ & 25 & 31,65 & 19 & 34,55 & & 1,08 & {$[0,68 ; 1,71]$} \\
\hline
\end{tabular}

Legenda: ${ }^{1}$ Teste Qui-Quadrado; ${ }^{2}$ Teste G de Williams;RR - Risco relativo; IC [95\%] - Intervalo com 95\% de confiança; CST - Cesareana Segmentar Transversa; PV - Parto Vaginal.

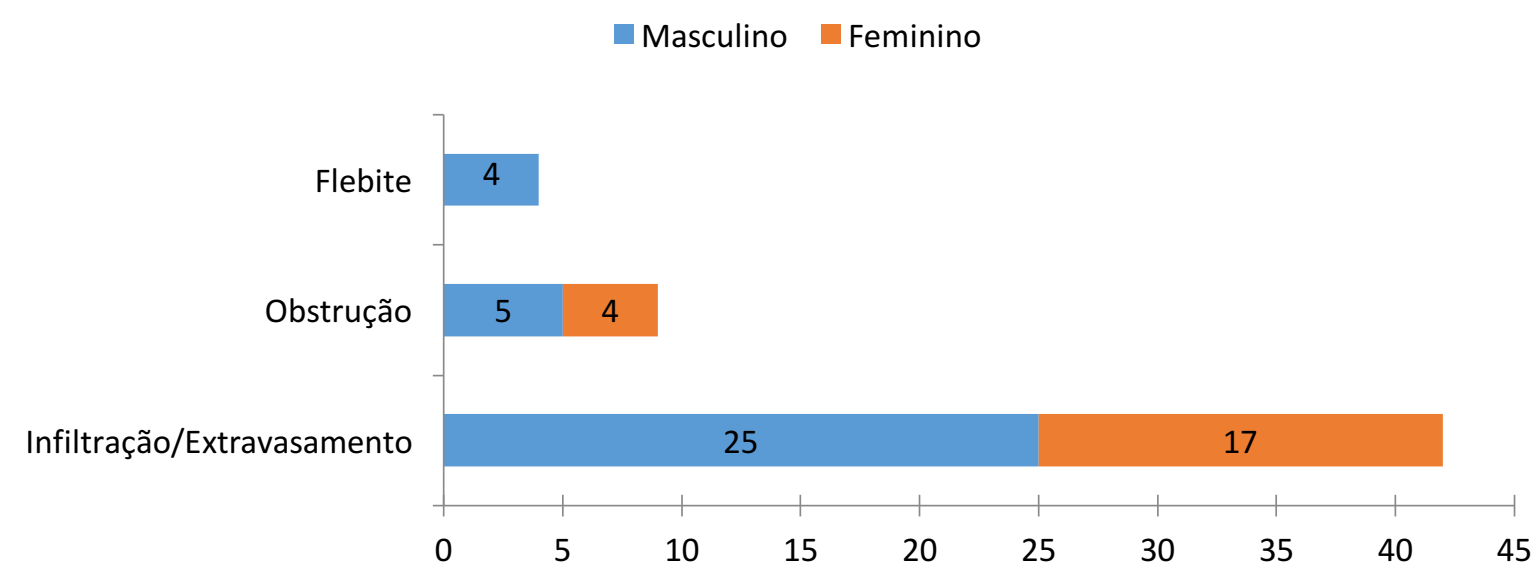

Figura 1: Distribuição dos cateteres com complicação de acordo com o sexo do neonato. Curitiba, PR, Brasil, 2013. 
Tabela 2: Análise das variáveis relacionadas ao cateter e seu uso associadas à ocorrência de complicações ( $n=134)$. Curitiba, PR, Brasil, 2013.

\begin{tabular}{|c|c|c|c|c|c|c|c|}
\hline \multirow{3}{*}{ Variável } & \multicolumn{4}{|c|}{ Complicação } & \multirow{3}{*}{ p-valor } & \multirow{3}{*}{$\mathbf{R R}$} & \multirow{3}{*}{ IC [95\%] } \\
\hline & \multicolumn{2}{|c|}{ Sim } & \multicolumn{2}{|c|}{ Não } & & & \\
\hline & $n=55$ & $\%$ & $n=79$ & $\%$ & & & \\
\hline \multicolumn{8}{|l|}{ Procedimento realizado por } \\
\hline Enfermeiro & 15 & 27,27 & 22 & 27,85 & 0,94161 & 1 & {$[0,64 ; 1,61]$} \\
\hline Auxiliar/Técnico & 40 & 72,73 & 57 & 72,15 & & 1,02 & \\
\hline \multicolumn{8}{|l|}{ Peso no dia da punção } \\
\hline$<1500$ & 11 & 20 & 17 & 21,52 & $0,9592^{1}$ & 0,97 & {$[0,55 ; 1,68]$} \\
\hline $1500<p<2500$ & 20 & 36,36 & 27 & 34,18 & & 1,05 & {$[0,66 ; 1,65]$} \\
\hline$>2500$ & 24 & 43,64 & 35 & 44,3 & & 1 & \\
\hline \multicolumn{8}{|l|}{ Membro puncionado } \\
\hline Cefálica & 1 & 1,82 & 2 & 2,53 & 0,59362 & 0,781 & {$[0,16 ; 3,92]$} \\
\hline MMSS & 50 & 90,91 & 67 & 84,81 & & 0,67 & - \\
\hline MMII & 4 & 7,27 & 10 & 12,66 & & & {$[0,28 ; 1,57]$} \\
\hline \multicolumn{8}{|l|}{ Tipo de infusão } \\
\hline Contínua & 23 & 41,82 & 40 & 50,63 & 0,29192 & 1 & \\
\hline Intermitente & 2 & 3,64 & 6 & 7,59 & & 0,68 & {$[0,20 ; 2,37]$} \\
\hline Ambas & 30 & 54,55 & 33 & 41,77 & & 1,3 & {$[0,86 ; 1,98]$} \\
\hline \multicolumn{8}{|l|}{ CVC } \\
\hline Não & 46 & 83,64 & 68 & 86,08 & $0,6966^{1}$ & 1 & \\
\hline Sim & 9 & 16,36 & 11 & 13,92 & & 1,12 & {$[0,65 ; 1,90]$} \\
\hline \multicolumn{8}{|l|}{ CVD } \\
\hline Não & 54 & 98,18 & 76 & 96,2 & 0,52102 & 1 & \\
\hline Sim & 1 & 1,82 & 3 & 3,8 & & 0,6 & {$[0,11 ; 3,33]$} \\
\hline \multicolumn{8}{|l|}{ IOT } \\
\hline Não & 38 & 69,09 & 62 & 78,48 & $0,2192^{1}$ & 1 & \\
\hline Sim & 17 & 30,91 & 17 & 21,52 & & 0,72 & {$[0,50 ; 1,60]$} \\
\hline \multicolumn{8}{|l|}{ Cirurgia } \\
\hline Não & 53 & 96,36 & 78 & 98,73 & 0,40382 & 1 & {$[0,72 ; 3,77]$} \\
\hline Sim & 2 & 3,64 & 1 & 1,27 & & 1,65 & \\
\hline \multicolumn{8}{|l|}{ Plano básico } \\
\hline Não & 3 & 5,45 & 7 & 8,86 & 0,24912 & 1 & \\
\hline Sim & 31 & 56,36 & 33 & 41,77 & & 1,61 & {$[0,61 ; 4,30]$} \\
\hline Uso exclusivo & 21 & 38,18 & 39 & 49,37 & & 1,17 & {$[0,43 ; 3,20]$} \\
\hline \multicolumn{8}{|l|}{ NPT } \\
\hline Não & 51 & 92,73 & 71 & 89,87 & 0,57342 & 1 & \\
\hline Sim & 4 & 7,27 & 8 & 10,13 & & 0,8 & {$[0,35 ; 1,82]$} \\
\hline \multicolumn{8}{|l|}{ ATM } \\
\hline Não & 29 & 52,73 & 51 & 64,56 & 0,16961 & 1 & \\
\hline Sim & 26 & 47,27 & 28 & 35,44 & & 1,33 & {$[0,89 ; 1,98]$} \\
\hline \multicolumn{8}{|l|}{ Transfusão } \\
\hline Não & 52 & 94,55 & 75 & 94,94 & 0,92322 & 1 & \\
\hline Sim & 3 & 5,45 & 4 & 5,06 & & 1,05 & {$[0,43 ; 2,52]$} \\
\hline \multicolumn{8}{|l|}{ Outros } \\
\hline Não & 38 & 69,09 & 59 & 74,68 & $0,4763^{1}$ & 1 & \\
\hline Sim & 17 & 30,91 & 20 & 25,32 & & 1,17 & {$[0,76 ; 1,80]$} \\
\hline \multicolumn{8}{|c|}{ Tempo de permanência dos cateteres } \\
\hline$<48 \mathrm{~h}$ & 42 & 76,36 & 46 & 58,23 & 0,02961 & 1,69 & {$[1,01 ; 2,81]$} \\
\hline$>48 h$ & 13 & 23,64 & 33 & 41,77 & & 1 & \\
\hline
\end{tabular}

Legenda: ${ }^{1}$ Teste Qui-Quadrado; ${ }^{2}$ Teste G de Williams; RR - Risco relativo; IC [95\%] - Intervalo com 95\% de confiança; Membros superiores (MMSS); Membros inferiores (MMII); Cateter venoso central (CVC); Cateter vesical de demora (CVD); Intubação orotraqueal (IOT); Nutrição parenteral total (NPT); Antimicrobiano (ATM). 


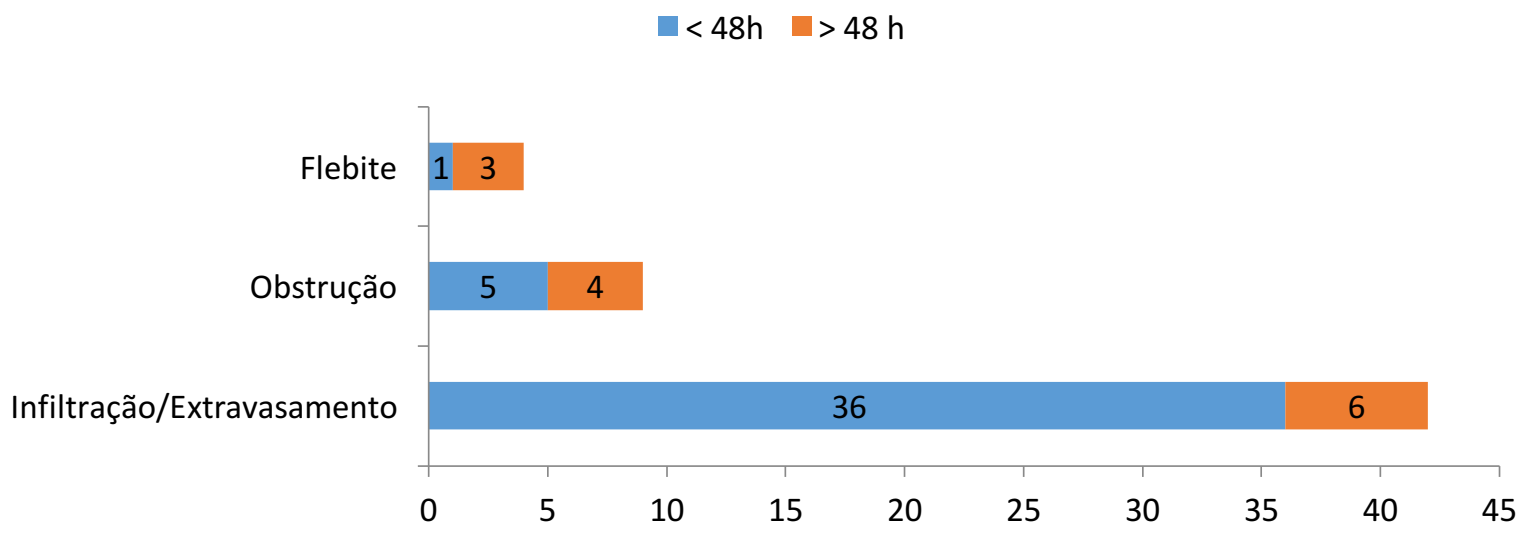

Figura 2: Distribuição dos cateteres com complicação de acordo com o motivo de retirada e o tempo de permanência. Curitiba, PR, Brasil, 2013.

\section{DISCUSSÃO}

A ocorrência de complicações como motivo de retirada dos CIP pesquisados apresenta valores inferiores $(41,04 \%)$ ao descrito pela literatura com população semelhante, a qual apresentava variabilidade entre 47,44 a $83 \%^{(8,13-15)}$.

Outros motivos de retirada observados foram retirada eletiva $(20,90 \%)$ e, com menor frequência, retirada acidental (5,97\%). Cateteres com retirada eletiva apresentaram-se em porcentagem mais satisfatória quando comparado a dados da literatura que descrevem percentagem de $18 \%$ a $24 \%{ }^{(8,15)}$. A literatura apresenta que $14 \%$ dos cateteres tiveram retirada acidental, valores inferiores aos descritos nesta pesquisa $^{(8)}$.

Dentre as complicações relacionadas ao uso do CIP, predominou infiltração/extravasamento (76,36\%), seguido de obstrução (16,36\%) e flebite (7,27\%). Em estudo desenvolvido com 36 recém-nascidos internados na UTIN de uma maternidade pública do Sudeste Brasileiro, cujo objetivo foi descrever a ocorrência de flebite, infiltração e extravasamento, verificou-se predominância do motivo de remoção do dispositivo intravenoso periférico infiltração com $79,2 \%$, seguido por $16,7 \%$ casos de flebite ${ }^{(8)}$. Na presente pesquisa o valor da complicação infiltração/extravasamento assemelha-se ao estudo supracitado, porém, ao comparar os casos de flebite, os achados dessa pesquisa foram inferiores.

Concernente à infiltração/extravasamento (76,36\%), considerou-se elevada a porcentagem constatada nesta pesquisa ao confrontar a estudos nacionais e internacionais ${ }^{(13-16)}$, que apresentam valores entre 25 e $56 \%$.

Os neonatos apresentam-se propensos à ocorrência de infiltração/extravasamento, devido características de flexibilidade do tecido subcutâneo, ocorrendo distensão deste com a presença do líquido, e ainda, por possuir integridade venosa prejudicada, facilitando a fuga capilar. Características, tais como fisiologia dos neonatos e fragilidade clínica desta clientela, são fatores que predispõem o desenvolvimento das complicações infiltração e extravasamento ${ }^{(17)}$.

Os fluídos com determinadas características aumentam o risco de extravasamento, são elas: 
osmolaridade (número de partículas por quilograma de solvente); vasoatividade (capacidade de causar constrição do vaso); citotoxicidade (capacidade de provocar danos celulares ou morte) e extremo de potencial de Hidrogênio $(\mathrm{pH})$ (inferior a cinco ou superior a nove). Desconsiderando a solução administrada, tem-se a alteração das características em função da concentração do medicamento e do diluente utilizado na preparação da mistura intravenosa, acarretando danos celulares ou morte do tecido do vaso ${ }^{(18)}$.

Independente da solução infundida, a vigilância deve ser recorrente, pois a infiltração, mesmo de solução fisiológica, pode resultar em sérios danos ao paciente ${ }^{(19)}$. Assim, reitera-se que a forma adequada para a prevenção de infiltração e extravasamento é a observação contínua do local de punção do cateter, no intuito de identificação precoce dos sinais, bem como intervenções imediatas após sua ocorrência.

Outra complicação observada nesta pesquisa foi a obstrução dos cateteres (16,36\%), a qual pode relacionar-se à precária manutenção da via de acesso após administração de medicamentos sem lavagem do cateter. Índices de complicação por obstrução do cateter descritos na literatura variam entre 7,1 a 39\% ${ }^{(14-15)}$, dado convergente ao encontrado nesta pesquisa.

Ao considerar a complicação flebite $(7,27 \%)$, observou-se porcentagem inferior ao descrito na literatura que variavam entre 9 e 16,7\% ${ }^{(8,14-15)}$. Esta complicação provém de fatores: químicos, relacionados às características das soluções infundidas; mecânicos, acarretados por movimentos contínuos desta clientela que são de difícil controle; ou infecciosos, caracterizados pelas práticas errôneas de manipulação dos cateteres.

Visando a prevenção de flebites, há práticas que devem ser contempladas durante punção e manipulação dos dispositivos intravenosos, quais sejam: higiene das mãos, seja por lavagem das mãos com água e sabão em técnica convencional, ou friccionando com álcool 70\%, e na pronta remoção do cateter em presença de sinais flogísticos ${ }^{(9)}$; bem como, a identificação precoce do surgimento desta complicação com a prática de vigilância constante do sítio de inserção do $\mathrm{CIP}^{(19)}$.

De modo geral, ao evitar o surgimento das complicações relacionadas ao uso do CIP, aplicam-se intervenções antes, durante e após a cateterização periférica. Porém, reforça-se como fator importante o conhecimento dos profissionais de enfermagem, por tratar-se dos funcionários que realizam cuidados contínuos e ininterruptos aos neonatos, bem como por serem os responsáveis pela punção, manipulação e manutenção dos cateteres.

A educação é um dos pilares na formação dos profissionais de saúde, devido às complexas mudanças no ato de cuidar e inserção contínua de novas tecnologias ${ }^{(20)}$, o que gera lacunas no conhecimento dos profissionais, necessitando atualização constante. Para tanto, o profissional precisa basear-se nas evidências disponibilizadas por estudos e recomendações de especialistas, bem como guidelines e protocolos de intervenção e tratamento, cujo objetivo é reduzir complicações potencialmente sérias ${ }^{(5,19,21)}$.

A visualização contínua do sitio de inserção é o meio precoce para o reconhecimento dos sinais e sintomas das complicações relacionadas ao uso do CIP. Destaca-se que a presença do CIP em neonatos e crianças necessita de avaliação a cada hora do dispositivo intravenoso, mediante observação e palpação, 
visto tratar-se de clientela que não consegue prover informações ${ }^{(22)}$.

Destarte, a fixação deve ser removida ao apresentar possíveis sinais clínicos de infecção, tal ação objetiva a visualização adequada do sítio de inserção do dispositivo ${ }^{(9,22)}$. Nos casos de CIP salinizado, o local deve ser avaliado no mínimo duas vezes por dia $^{(22)}$. Tais intervenções podem minimizar dor e sofrimento que as complicações provenientes da TIV acarretam aos neonatos.

Concernentes ao CIP, as variáveis clínicas e epidemiológicas relacionadas ao neonato (tipo de parto, motivo de internação, Apgar no primeiro e quinto minuto, Parkin, peso ao nascer) não se apresentaram como fatores de risco. A única variável epidemiológica que apresentou maior propensão para desenvolvimento de complicação foi o sexo, masculino $(p=0,0152 ; R R=1)$. Relacionado ao uso do $C I P$, evidenciou-se que nas primeiras 48 horas após a punção do cateter ocorre o maior risco de desenvolver complicação $(p=0,0296$, $R R=1,69$ ). Esse período corresponde igualmente às primeiras 48 horas de vida do neonato, pois ao nascer são encaminhados, quando necessário, diretamente à UTIN, a qual tem como rotina o cateterismo periférico no momento do internamento. Portanto, as condutas adotadas quanto à vigilância do CIP devem ser modificadas principalmente nas primeiras 48 horas de vida, quando o neonato apresenta instabilidade hemodinâmica e observou-se o aumento no desenvolvimento de complicações. Após ser submetido ao procedimento da terapia intravenosa, o neonato que se apresenta clinica e fisiologicamente fragilizado recebe terapêutica que estabiliza e equilibra as condições gerais, motivo que pode explicar a diminuição de complicações após esse período.

\section{CONCLUSÕES}

A incidência de ocorrência de complicações relacionadas ao primeiro CIP em neonatos hospitalizados em UTIN foi alta. Dentre as complicações observou-se maior incidência de infiltração/extravasamento, seguida de obstrução e flebite. As variáveis que apresentaram significância estatística para o desenvolvimento de complicações foram o sexo masculino e o período de 48 horas após a punção.

A terapia intravenosa periférica é de suma importância para o cuidado a neonatos críticos e a equipe de enfermagem é responsável pelos procedimentos envolvidos com esta tecnologia. As evidências resultantes desta pesquisa embasam um cuidado de qualidade, beneficiando a população em estudo.

Fatores limitantes desta pesquisa relacionam-se aos registros incompletos quanto ao motivo de retirada dos cateteres, bem como ausência de informações para determinar o grau das complicações.

O desenvolvimento de estudos semelhantes para contemplar graus de flebite, infiltração e extravasamento, bem como a comparação de riscos e benefícios com outras tecnologias de cateter intravenoso periférico, são necessários para gerar melhores evidências no cuidado em enfermagem neonatal.

\section{REFERÊNCIAS}

1. Piccoli A, Soares CRS, Costa G, Silveira JL, Fiatt MP, Cunha RS. Perfil clínico de neonatos de muito baixo peso 
internados em uma Unidade de Tratamento Intensivo Neonatal Clinical \& Biomedical Research [Internet]. 2013 [acesso em: 31 mar. 2016];32(4):412-9. Disponível em: http://seer.ufrgs.br/index.php/hcpa/article/view/31904. 2. Bonnici ET. Safer patient care through better Peripheral Intravenous Catheter management. International Journal of Infection Control [Internet]. 2012 [acesso em: 31 mar. 2016];8(2). Disponível em:

http://www.ijic.info/article/view/9074.

3. Infusion Nurses Society. Infusion nursing standarts of pratice. J Infus Nurs [Internet]. 2011 [acesso em: 31 mar. 2016];34(1S). Disponível em:

http://www.ins1.org/files/public/11_30_11_Standards_of_Practice_2011_Cover_TOC.pdf.

4. Menezes SO, Gomes MASM, Lamy Filho F. Manejo do acesso vascular em recém-nascidos de muito baixo peso ao nascer em unidades públicas neonatais do município do Rio de Janeiro. Revista de Pesquisa em Saúde [Internet]. 2013 [acesso em: 31 mar. 2016];14(1):11-5. Disponível em:

http://www.periodicoseletronicos.ufma.br/index.php/revistahuufma/article/view/1717.

5. Simona R. A Pediatric Peripheral Intravenous Infiltration Assessment Tool. J Infus Nurs [Internet]. 2012 [acesso em: 31 mar. 2016];35(4):243-8. Disponível em: http://dx.doi.org/10.1097/NAN.0b013e31825af323.

6. Perez A, Feuz I, Brotschi B, Bernet V. Intermittent flushing improves cannula patency compared to continuous infusion for peripherally inserted venous catheters in newborns: results from a prospective observational study. J Perinat Med [Internet]. 2012 [acesso em: 31 mar. 2016];40(3):311-4. Disponível em: http://dx.doi.org/10.1515/jpm2011-1000.

7. Phillips LD. Manual of I.V. Therapeutics Evidence-Based Practice for Infusion Therapy. 5a ed. Philadelphia: F. A. Davis Company; 2010.

8. Gomes ACR, Silva CAG, Gamarra CJ, Faria JCO, Avelar AFM, Rodrigues EC. Assessment of phlebitis, infiltration and extravasation events in neonates submitted to intravenous therapy. Esc Anna Nery [Internet]. 2011 [acesso em: 31 mar. 2016];15(3):472-9. Disponível em: http://dx.doi.org/10.1590/S1414-81452011000300005.

9. O'Grady NP, Alexander M, Dellinger EP, Gerberding JL, Heard SO, Maki DG, et al. Guidelines for the prevention of intravascular catheter-related infections. Centers for Disease Control and Prevention. MMWR Recomm Rep [Internet]. 2002 [acesso em: 31 mar. 2016];51(RR-10):1-29. Disponível em: http://www.cdc.gov/mmwr/PDF/rr/rr5110.pdf.

10. Modes PSSA, Gaíva MAM, Rosa MKO, Granjeiro CF. Cuidados de enfermagem nas complicações da punção venosa periférica em recém-nascidos. Rev Rene [Internet]. 2011 [acesso em: 31 mar. 2016];12(2):324-32. Disponível em: http://www.revistarene.ufc.br/revista/index.php/revista/article/view/160.

11. Higginson R, Parry A. Phlebitis: treatment, care and prevention. Nurs Times [Internet]. 2011 [acesso em: 31 mar. 2016];107(36):18-21. Disponível em: http://www.nursingtimes.net/clinical-archive/infection-control/phlebitistreatment-care-and-prevention/5034782.fullarticle.

12. Lanzillotti LS, Seta MH, Andrade CLT, Mendes Junior WV. Adverse events and other incidents in neonatal intensive care units. Ciênc. saúde coletiva [Internet]. 2015 [acesso em: 31 mar. 2016];20(3):937-46. Disponível em:

http://dx.doi.org/10.1590/1413-81232015203.16912013.

13. Perez JMJ, Villanueva SG, Rodríguez LR, Llarena RMR. Utilización y mantenimiento de los catéteres venosos periféricos en la unidad de neonatología del Hospital Universitario Río Hortega, Valladolid. Rev Enfermería CyL [Internet]. 2015 [acesso em: 31 mar. 2016];7(1):3-11. Disponível em:

http://www.revistaenfermeriacyl.com/index.php/revistaenfermeriacyl/article/view/143.

14. Arnts IJ, Heijnen JA, Wilbers HT, van der Wilt GJ, Groenewoud JM, Liem KD. Effectiveness of heparin solution versus normal saline in maintaining patency of intravenous locks in neonates: a double blind randomized controlled study. J Adv Nurs [Internet]. 2011 [acesso em: 31 mar. 2016];67(12):2677-85. Disponível em:

http://dx.doi.org/10.1111/j.1365-2648.2011.05718.x.

15. Hetzler R, Wilson M, Hill EK, Hollenback C. Securing Pediatric Peripheral IV Catheters-Application of an EvidenceBased Practice Model. J Pediatr Nurs [Internet]. 2011 [acesso em: 31 mar. 2016];26(2):143-8. Disponível em: http://dx.doi.org/10.1016/j.pedn.2010.12.008.

16. Barbosa MTSR, Alves VH, Rodrigues DP, Branco MBLR, Souza RMP, Bonazzi VCAM. Quality indicators in support of intravenous therapy in a university hospital: a contribution of nursing. Rev. pesqui. cuid. fundam. (Online) [Internet]. 2015 [acesso em: 31 mar. 2016];7(2):2277-86. Disponível em: http://dx.doi.org/10.9789/2175-5361.2015.v7i2.2277$\underline{2286 .}$.

17. Wu J, Mu D. Vascular catheter-related complications in newborns. J Paediatr Child Health [Internet]. 2012 [acesso 
em: 31 mar. 2016];48(2):E91-5. Disponível em: http://dx.doi.org/10.1111/j.1440-1754.2010.01934.x

18. Clark E, Giambra BK, HingI J, Doellman D, Tofani B, Johnson N. Reducing risk of harm from extravasation: a 3-tiered evidence-based list of pediatric peripheral intravenous infusates. J Infus Nurs [Internet]. 2013 [acesso em: 31 mar.

2016];36(1):37-45. Disponível em: http://dx.doi.org/10.1097/NAN.0b013e3182798844.

19. Oliveira ASS, Parreira PMSD. Intervenções de enfermagem e flebites decorrentes de cateteres venosos periféricos. Revisão sistemática da literatura. Rev Enferm Ref [Internet]. 2010 [acesso em: 31 mar. 2016];serlII(2):137-47.

Disponível em: http://www.scielo.mec.pt/scielo.php?script=sci_arttext\&pid=S0874-

02832010000400015\&lng=pt\&nrm=iso\&tlng=pt.

20. Castral TC, Daré MF, Scochi CGS. Prioridades de pesquisa em enfermagem neonatal e pediátrica. Rev. Eletr. Enf. [Internet]. 2014 [acesso em: 31 mar. 2016];16(1):12-4. Disponível em: http://dx.doi.org/10.5216/ree.v16i1.29266. 21. Harpel J. Best practices for vascular resource teams. J Infus Nurs [Internet]. 2013 [acesso em: 31 mar. 2016];36(1):46-50. Disponível em: http://dx.doi.org/10.1097/NAN.0b013e3182798862.

22. Gorski LA, Hallock D, Kuehn SC, Morris P, Russell JM, Skala LC. Recommendations for Frequency of Assessment of the Short Peripheral Catheter Site. J Infus Nurs [Internet]. 2012 [acesso em: 31 mar. 2016];35(5):290-2. Disponível em: http://dx.doi.org/10.1097/NAN.0b013e318267f636. 